Skin

Pharmacology

and

Physiology
Skin Pharmacol Physiol 2020;33:170-177

DOI: $10.1159 / 000508344$
Received: February 4, 2020

Accepted: April 30, 2020

Published online: June 22, 2020

\title{
Vascular Endothelial Growth Factor Blockade Induces Dermal Endothelial Cell Apoptosis in a Clinically Relevant Skin Organ Culture Model
}

\author{
Andrea Luengas-Martinez ${ }^{\mathrm{a}}$ Jonathan Hardman-Smart ${ }^{\mathrm{a}, \mathrm{b}}$ David Rutkowski ${ }^{\mathrm{a}}$ \\ Talveen S. Purba ${ }^{\mathrm{a}}$ Ralf Paus ${ }^{\mathrm{a}, \mathrm{c}}$ Helen S. Young ${ }^{\mathrm{a}}$ \\ ${ }^{a}$ Centre for Dermatology Research and Manchester Academic Health Science Centre, The University of Manchester, \\ Manchester, UK; ' St John's Institute for Dermatology, King's College London, London, UK; 'Department of \\ Dermatology and Cutaneous Surgery, School of Medicine, University of Miami, Miami, FL, USA
}

\section{Keywords}

Angiogenesis · Psoriasis · Vascular biology · Organ culture

\begin{abstract}
Background/Aims: Vascular endothelial growth factor (VEGF), a key mediator of angiogenesis, plays a key role in physiological processes and is a major contributor to several diseases including cancer and psoriasis. Anti-VEGF therapies are widely used as cancer and ophthalmological treatments. There is some evidence that VEGF blockade may have utility in the management of psoriasis, although their potential has been largely unexplored. We hypothesized that a human skin organ culture could provide a stable ex vivo model in which the cutaneous microvascular network could be studied and experimentally manipulated. Methods: Punch biopsies $(3 \mathrm{~mm}$ ) of skin, donated by healthy individuals (39-72 years old, $n=5$ ), were incubated with monoclonal antibody (mAb) to human VEGF (bevacizumab) at doses based on data from animal and clinical studies. After 3-day culture, cell death and proliferation as well as vascular endothelial cell changes were assessed using quantitative immunohistomorphometry. Results: Anti-VEGF mAb at $0.8 \mathrm{mg} / \mathrm{mL}$ in-
\end{abstract}

duced a significant increase in cleaved caspase- 3 expression in $\mathrm{CD}_{3} 1^{+}$cells $(p<0.05)$. None of the doses tested increased TUNEL or decreased Ki-67 expression in the basal layer of the epidermis, confirming the model's viability. In addition, the lactate dehydrogenase (LDH) assay showed no increase in LDH activity in treated samples compared to untreated control. The highest anti-VEGF $\mathrm{mAb}$ dose $(0.8 \mathrm{mg} / \mathrm{mL})$ induced an increase in TUNEL expression in the upper epidermis, which did not correlate with caspase-3 immunoreactivity. Further investigation revealed that anti-VEGF mAb did not change the expression of markers of terminal differentiation such as keratin 10, filaggrin, and involucrin, suggesting that VEGF depletion does not affect keratinocyte terminal differentiation. In contrast to the control group, levels of VEGF protein were undetectable in the culture supernatant of samples treated with $0.8 \mathrm{mg} / \mathrm{mL}$ of anti-VEGF mAb, suggesting sufficient dose. Conclusion: Our pilot study provides the first evidence that anti-VEGF therapy promotes endothelial cell apoptosis in human skin ex vivo. Our pragmatic human skin organ culture assay offers a valuable tool for future preclinical endothelial cell and translational microvascular network/anti-angiogenesis research in human skin.

(c) 2020 S. Karger AG, Basel

\section{KARGER}

(C) 2020 S. Karger AG, Basel

karger@karger.com

www.karger.com/spp
Andrea Luengas-Martinez

The University of Manchester

Centre for Dermatology Research and Manchester Academic Health Science Centre

1.823 Stopford Building, Manchester M13 9PT (UK)

andrea.luengasmartinez@manchester.ac.uk 


\section{Introduction}

Angiogenesis, the formation and remodelling of new blood vessels from a pre-existing vascular bed, plays a key role in physiological processes such as wound healing. Angiogenesis is a major contributor to pathological conditions including cancer, eye diseases, and psoriasis [1-3]. Vascular endothelial growth factor (VEGF), a key mediator of angiogenesis, activates signalling pathways which promote endothelial cell proliferation, migration, and survival as well as vasodilation and vascular permeability [4]. Anti-VEGF therapy has been used to target pathological angiogenesis in the treatment of oncological and ophthalmological diseases for over 15 years [5]. There are a small number of case reports of clearance of psoriasis in patients with the disease who were being treated for malignancy with VEGF inhibitors $[6,7]$. However, the effects of systemic anti-VEGF therapy on human skin remain poorly characterized, and the potential of anti-VEGF in the management of selected dermatological diseases such as psoriasis merits further investigation.

To achieve this, readily available and clinically relevant preclinical research assays are required, with full-thickness serum-free human skin organ culture providing one pragmatic option. These simple and experimentally tractable ex vivo assays are more likely to reflect relevant cellcell, cell-matrix, and tissue interactions in human skin in situ than $3 \mathrm{D}$ or $2 \mathrm{D}$ angiogenesis assay systems [8-10]. Most importantly, utilizing organ-cultured skin biopsies from dermatological conditions such as psoriasis offers the opportunity to interrogate the angiogenic effect of xenobiotic molecules in a clinically relevant preclinical model.

Therefore, we hypothesized that a human skin organ culture could provide a stable ex vivo model in which the cutaneous microvascular network could be studied and experimentally manipulated. As a first step towards this goal, we wished to obtain proof-of-principle that antiVEGF therapeutics can be instructively studied in organcultured healthy human skin. Therefore, we established an organ culture model and used an IgG $_{1}$ humanized monoclonal antibody (mAb) directed against all VEGF isoforms (bevacizumab, Avastin ${ }^{\circledR}$, Roche). Bevacizumab was the first anti-VEGF mAb licensed for any disease indication and is licensed to be used for age-related macular degeneration (AMD) and metastatic cancers of the colon, breast, kidney, lung, and ovary $[5,11]$.

VEGF Inhibition in Human Skin Culture

\section{Materials and Methods}

\section{Tissue Collection and Ethics}

Human skin samples were obtained through the Manchester Skin Health Biobank (19/NW/0082). Human tissue collection and handling was performed according to the "Human Tissue Act guidelines" with approval from the institutional research ethics committee. Written informed consent was obtained from the study volunteers, who were recruited from Salford Royal NHS Foundation Trust, Manchester, UK.

Our study included samples from 5 healthy volunteers ( 5 biological replicates) aged from 39 to 72 (average 50.2) and 4 biopsies per volunteer. Skin biopsies ( $3 \mathrm{~mm}$ ) were taken from the donated skin samples, and the excess adipose tissue was removed. Skin was cultured overnight at $37^{\circ} \mathrm{C}$ with $5 \% \mathrm{CO}_{2}$ to adapt to culture conditions, after which the media was replaced and the drug conditions were added. The replicates required were iterated from previous studies by our group so that the individual assays were adequately powered [12].

In addition, samples from the uninvolved skin ( $5 \mathrm{~cm}$ away from the plaque) of 1 patient with psoriasis (aged 19) were used in a proof-of-concept extension study to confirm the potential of our model to be utilized in a disease context such as psoriasis.

\section{Human Skin Organ Culture}

Samples were cultured in $1 \mathrm{~mL}$ of serum-free supplemented William's E media (Gibco ${ }^{\circledR}$, Thermo Fisher Scientific, UK) [13] with mAb for human VEGF (bevacizumab, Avastin ${ }^{\circledR}$ ) for 3 days. Skin biopsies incubated with supplemented William's E medium (Gibco) served as controls. In all cultures, the samples were orientated with the epidermis facing upwards at the liquid-air interface and the dermis submerged in the media.

The medium and supplements used are based in the serum-free media described by Philpott et al. [14]. This medium contains William's E medium (Gibco) supplemented with $2 \mathrm{~mm}$ of l-glutamine (Invitrogen $^{\mathrm{TM}}$, Paisley, UK), $10 \mathrm{ng} / \mathrm{mL}$ of hydrocortisone (SigmaAldrich $^{\circledR}$ ), $10 \mu \mathrm{g} / \mathrm{mL}$ of insulin (Sigma-Aldrich ${ }^{\circledR}$ ), and $1 \mu \mathrm{g} / \mathrm{mL}$ of penicillin/streptomycin antibiotic mixture (Sigma-Aldrich $\left.{ }^{\circledR}\right)$. An isotype control was not used in these pilot investigations as previous studies have determined the neutralizing activity of bevacizumab against VEGF is specific [15].

\section{Bevacizumab Treatment}

Bevacizumab is usually administered intravenously in concentrations ranging from 5 to $15 \mathrm{mg} / \mathrm{kg}$ [16-18]. The healthy skin biopsies were incubated with anti-VEGF mAb (bevacizumab, Avas$\left.\operatorname{tin}^{\circledR}\right)$ at doses based on human intravenous doses and serum concentrations observed in human and animal studies and these were $0.01,0.2$, and $0.8 \mathrm{mg} / \mathrm{mL}$. The media was changed every $48 \mathrm{~h}$ as described by Philpott et al. [14]. Skin biopsies from the uninvolved skin of a patient with psoriasis were incubated with anti-VEGF $\mathrm{mAb}$ at $0.8 \mathrm{mg} / \mathrm{mL}$. After skin organ culture for the indicated time, tissue was embedded for cryosectioning and immunohistochemistry.

\section{Immunofluorescence Staining}

Skin samples were embedded in OCT compound (KP-CryoCompound Frozen Tissue Medium). Non-serial 8- $\mu \mathrm{m}$ cryosections were taken using a cryostat (Cryostat Bright OTF, 5000-001) and affixed to Superfrost Plus Micro slides (Menzel Glaser ${ }^{\mathrm{TM}}$, 
Thermo-Scientific). Cell death was investigated using the terminal deoxynucleotidyl transferase (TdT) dUTP Nick-End Labeling (TUNEL; ApopTag ${ }^{\circledR}$ Plus Fluorescein in situ kit, Millipore) assay, and $\mathrm{Ki}-67$ was used to assess proliferation. An antibody against the pan-endothelial marker CD31 in combination with an antibody against the lymphatic-specific hyaluronan receptor (LYVE-1) was used to study CD $31^{+}$LYVE- $1^{-}$blood vessel endothelial cells. Endothelial cell apoptosis and proliferation were investigated by double immunofluorescence using antibodies against CD31-cleaved caspase- 3 and CD31/Ki-67, respectively. Keratin 10, filaggrin, and involucrin were used to investigate keratinocyte terminal differentiation. Primary antibodies used were mouse CD31 (1:100, Dako; JC/70A), rabbit LYVE-1 (1:100, Abcam; ab102278), rabbit cleaved caspase-3 (1:50, Cell Signalling, Boston, MA, USA; Asp175), rabbit Ki-67 (1:50, Abcam; ab16667), rabbit keratin 10 (1:150, Abcam; ab97764), rabbit filaggrin (1:250, Abcam; ab81468), and mouse involucrin (1:50; Sigma-Aldrich; SY5). Secondary antibodies used were goat anti-rabbit $488 \mathrm{AF}$ (1:200), goat anti-rabbit $594 \mathrm{AF}$ (1:200), and goat anti-mouse 594 AF (1:200). After staining, slides were mounted using fluoromount mounting medium (Dako; S3023) and were visualized and photographed the following day at $\times 200$ and $\times 400$ magnification using Keyence Biozero 8000 (Keyence Corporation).

\section{Analysis of the Blood Vessels}

Computer-assisted morphometric analysis of blood vessels was performed using a macro for ImageJ (Fiji) to analyze the average dermal blood vascular surface area. The macro can be found in the following repository: https://github.com/gadeamm/MacroAnalysisBloodVessels. The number of CD $31^{+} \mathrm{LYVE}-1^{-}$cells with visible nuclei $\left(\mathrm{DAPI}^{+}\right)$per visual field were counted with ImageJ (Fiji) and used as an internal control for the macro.

\section{Immunoassay for VEGF Detection}

A sandwich ELISA (Quantikine ELISA, R\&D Systems, Abingdon, UK) was performed to measure the levels of VEGF $_{165}$ protein in the tissue culture supernatant according to the manufacturer's instructions [19]. The limit of the detectable $\mathrm{VEGF}_{165}$ concentration was $5 \mathrm{pg} / \mathrm{mL}$. Samples and standards were added to anti-human VEGF pre-coated 96-well plates and incubated for $2 \mathrm{~h}$ at RT followed by two washing steps using Wash Buffer (Quantikine ELISA, R\&D Systems). Next, human VEGF Conjugate (Quantikine ELISA, R\&D Systems) was added to each well and incubated

Fig. 1. The effect of anti-VEGF mAb in human skin organ culture. a Endothelial cell death was assessed using CD31/cleaved caspase- 3 double labelling. DAPI was used for nuclear staining. $\mathbf{b}$ The red arrows indicate $\mathrm{CD} 31^{+}$endothelial cells and green arrows indicate $\mathrm{CD} 31^{+}$cleaved caspase- $3^{+}$endothelial cells. Anti-VEGF $\mathrm{mAb}$ at $0.8 \mathrm{mg} / \mathrm{mL}$ significantly upregulated cleaved caspase $-3^{+}$ $\mathrm{CD} 31^{+}$cells in skin organ culture. $\mathrm{c}$ Anti-VEGF mAb did not affect cleaved caspase-3 expression in the stratum granulosum (SG). d The skin average blood vessel surface area and the number of blood vessel endothelial cells were assessed using CD31/LYVE-1 double labelling. The white broken line indicates the epidermaldermal junction. The red arrows indicate CD $31^{+}$cells and green arrows indicate LYVE- $1^{+}$endothelial cells. e Anti-VEGF mAb did for $2 \mathrm{~h}$ at RT followed by a washing step. Next, substrate solution was added to each well and incubated for $20 \mathrm{~min}$ at RT followed by Stop Solution (Quantikine ELISA, R\&D Systems), and optical density was measured at $450 \mathrm{~nm}$ with a correction at $550 \mathrm{~nm}$ using a microplate reader (CLARIOstar, BMG Labtech). All samples and standards were assayed in duplicate. A standard curve was prepared with VEGF ranging from $15.6 \mathrm{pg} / \mathrm{mL}$ to $1,000 \mathrm{pg} / \mathrm{mL}$.

\section{Lactate Dehydrogenase Assay}

Tissue toxicity was assessed using lactate dehydrogenase (LDH) fluorometric assay (Abcam; ab197000) according to the manufacturer's instructions. Samples, standards, and the positive control were added to the wells. A reaction mix containing $\mathrm{LDH}$ assay buffer, PicoProbe, and LDH substrate mix was added to each well, and fluorescence was measured immediately in kinetic mode every $2 \mathrm{~min}$ at $535 / 587 \mathrm{~nm}$ at $37^{\circ} \mathrm{C}$ for $10 \mathrm{~min}$.

\section{Statistical Analysis}

All statistical analyses and graphs were generated using Graphpad Prism 7.04 (Graph Pad Software, Inc., San Diego, CA, USA). All data presented are given as mean \pm SD. One-way ANOVA was used to analyze the differences between the control and the treated groups. Appropriate post hoc comparisons were used as indicated in figure legends, and $p<0.05$ was regarded as significant.

\section{Results and Discussion}

Anti-VEGF mAb (concentration $0.8 \mathrm{mg} / \mathrm{mL}$ ) induced a significant increase in cleaved caspase-3 expressing $\mathrm{CD} 31^{+}$endothelial cells in the dermis, as measured by number of cleaved caspase- $3^{+} \mathrm{CD} 31^{+}$cells per field of view, after 3-day organ culture ( $p<0.05$; Fig. 1a, b). Lower doses of anti-VEGF $\mathrm{mAb}(0.01$ and $0.2 \mathrm{mg} / \mathrm{mL})$ did not exert this effect on human skin organ culture. This corresponds well to the observation that anti-VEGF $\mathrm{mAb}$ shortens the VEGF-mediated survival of cultured endothelial cells in vitro [10]. Thus, our simple human skin organ culture model is suited to study the impact of clinically relevant anti-VEGF test agents on the apoptosis of

not affect the average blood vessel surface area or the number of blood vessel endothelial cells. $f$ Skin viability was examined by Ki$67 / T U N E L$ double labelling. The percentage of positive cells was analyzed in the stratum basale. The red arrows indicate $\mathrm{Ki}-67^{+}$cells and green arrows indicate $\mathrm{TUNEL}^{+}$cells. $\mathbf{g}$ Anti-VEGF $\mathrm{mAb}$ did not affect the percentage of $\mathrm{Ki}-67^{+}$cells or $\mathrm{TUNEL}^{+}$cells in the stratum basale. Number of independent experiments $n=5$ different donors (i.e., 1 punch per patient and per treatment group and at least 7 photomicrographs were analyzed per condition). Scale bars $\mathbf{a}, \mathbf{f}=50 \mu \mathrm{m} ; \mathbf{d}=100 \mu \mathrm{m}$. Data were analyzed with one-way ANOVA, mean \pm SD (Tukey's multiple comparison test). VEGF, vascular endothelial growth factor; $\mathrm{mAb}$, monoclonal antibody. ${ }^{*} p<0.05$.

(For figure see next page.) 


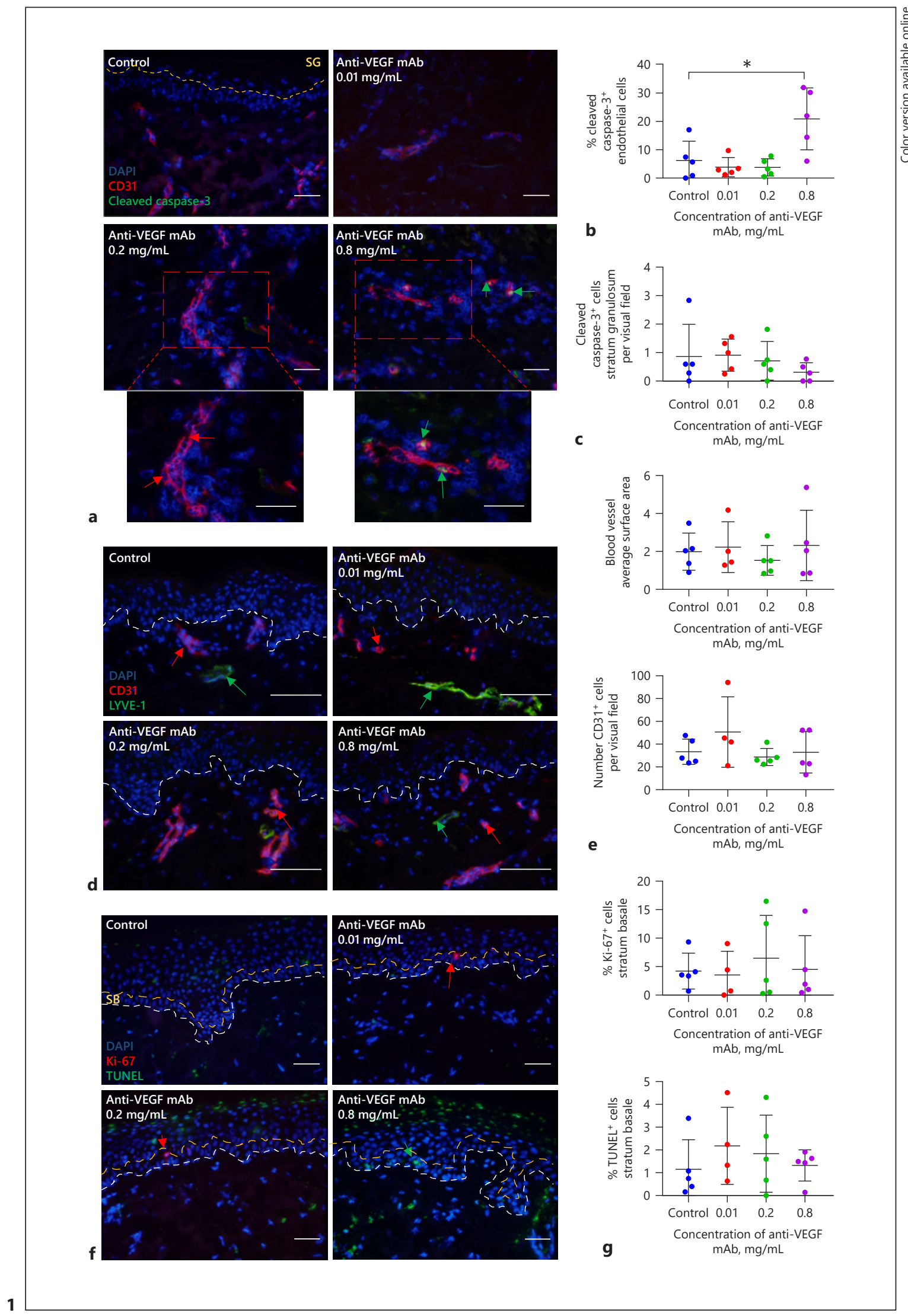


human skin endothelial cells within their physiological tissue habitat ex vivo.

Quantitative immunohistomorphometry of the blood vessels revealed that the average dermal blood vascular surface area or the number of blood vessel endothelial cells was not affected by VEGF blockade in 3-day culture (Fig. 1d, e), suggesting that overall blood vessel structure and density was maintained in our ex vivo human skin organ culture model. Moreover, anti-VEGF mAb did not affect endothelial cell proliferation as shown by CD31 and Ki-67 double labelling (online suppl. Fig. 1; see www. karger.com/doi/10.1159/000508344 for all online suppl. material). These results were expected as there is very little angiogenesis and endothelial cell proliferation in normal skin, but invite further study in skin diseases with increased/pathological angiogenesis, such as psoriasis.

In order to test the model viability, cell death and cell proliferation were studied in the stratum basale in the epidermis. Anti-VEGF mAb did not decrease the percentage of Ki-67 positive cells or increase terminal deoxynucleotidyl transferase (TdT) dUTP Nick-End Labeling (TUNEL) or cleaved caspase- 3 expression in the stratum basale of organ-cultured human skin as measured by quantitative immunohistomorphometry (Fig. 1f, g), thus confirming the expected epidermal viability under assay conditions [8]. Anti-VEGF $\mathrm{mAb}$ at $0.8 \mathrm{mg} / \mathrm{mL}$ induced an increase in TUNEL expression in the stratum granulosum ( $p<0.01$; online suppl. Fig. 2). Further investigation revealed no cleaved caspase- 3 expression, a specific marker for apoptosis, in the stratum granulosum (Fig. 1c) and normal expression of key keratinocyte terminal differentiation markers keratin 10, filaggrin, and involucrin (Fig. 2a, b). This suggests that anti-VEGF treatment did not alter keratinocyte survival, proliferation, or terminal differentiation within organ-cultured epidermis and thus primarily targeted intradermal endothelial cells during the incubation period. Levels of LDH enzyme were measured in the culture supernatant of samples treated with anti-VEGF mAb at $6,12,24$, and $48 \mathrm{~h}$. There was no increase in LDH activity in samples treated with anti-VEGF $\mathrm{mAb}$ compared to control, suggesting that anti-VEGF $\mathrm{mAb}$ was not toxic for human skin ex vivo at none of the doses tested (online suppl. Fig. 3).

Next, the levels of $\mathrm{VEGF}_{165}$ protein were quantified in the organ culture supernatant of skin biopsies incubated with $0.8 \mathrm{mg} / \mathrm{mL}$ of anti-VEGF $\mathrm{mAb}$ at different time points $(6,12,24$, and $48 \mathrm{~h})$ using an ELISA kit (ELISA; Quantikine ELISA, R\&D Systems) that detects free VEGF $_{165}$ but not VEGF bound to anti-VEGF mAb [16]. Levels of VEGF increased with time in the control sam- ples, whereas the levels of VEGF were undetectable (below the lower limit of detection $5 \mathrm{pg} / \mathrm{mL}$ ) in the samples treated with $0.8 \mathrm{mg} / \mathrm{mL}$ of anti-VEGF $\mathrm{mAb}$ at all time points tested (online suppl. Fig. 4). These results suggest that (1) VEGF is released from skin biopsies into the culture media gradually with time, reaching its highest concentration in the organ culture supernatant at $48 \mathrm{~h}$ and (2) anti-VEGF $\mathrm{mAb}$ at $0.8 \mathrm{mg} / \mathrm{mL}$ neutralizes $\mathrm{VEGF}_{165}$ released from the skin into the organ culture supernatant during culture. It is possible that the VEGF protein-drug complexes are still present in the treated biopsy samples. Alternatively, they may have been degraded within the tissue. Other investigators have suggested that complex degradation may occur through a variety of mechanisms such as pinocytosis and lysosomal degradation, cytosolic degradation, or degradation of internalized VEGF protein-drug complexes [20].

As a proof of concept, the effects of anti-VEGF mAb were tested in the uninvolved skin of 1 patient with psoriasis (online suppl. Fig. 5). Arguably, psoriasis pathogenesis is preferentially studied by utilizing uninvolved skin [21]. Differences in gene expression between uninvolved psoriatic skin and healthy control skin have been shown, suggesting similarity to skin from plaques of psoriasis [22]. Others have shown $\mathrm{CD} 57^{+} \mathrm{T}$ cells are less frequent in lesional compared to non-lesional skin due to the high cell turnover [23]. Our images show visible induction of endothelial cell apoptosis and the viability of the skin was not affected, demonstrating that our model could be used in a psoriasis context to study the effects of anti-VEGF $\mathrm{mAb}$ in the skin in situ.

Other angiogenic factors such as angiopoietin 1 and 2 as well as thrombospondin- 1 also participate in the regulation of the microvascular network and affect endothelial cell function. However, these factors act on the blood vasculature and affect endothelial cells using different physiological mechanisms and activating different signalling pathways [24]. It was outside the scope of this study to look into alternative cellular pathways that act on the dermal vasculature.

In conclusion, our pilot study provides the first evidence that anti-VEGF therapy promotes endothelial cell apoptosis in human skin ex vivo. In addition, we introduce a simple human skin organ culture model, which is reproducible and reliant only on the availability of human skin. In our model, the effects of anti-VEGF therapeutics on the apoptosis of native human vascular endothelial cell can be instructively studied within intact human dermis. The assay also permits assessment of human blood vessel density and structure. Moreover, endothelial cells in this 

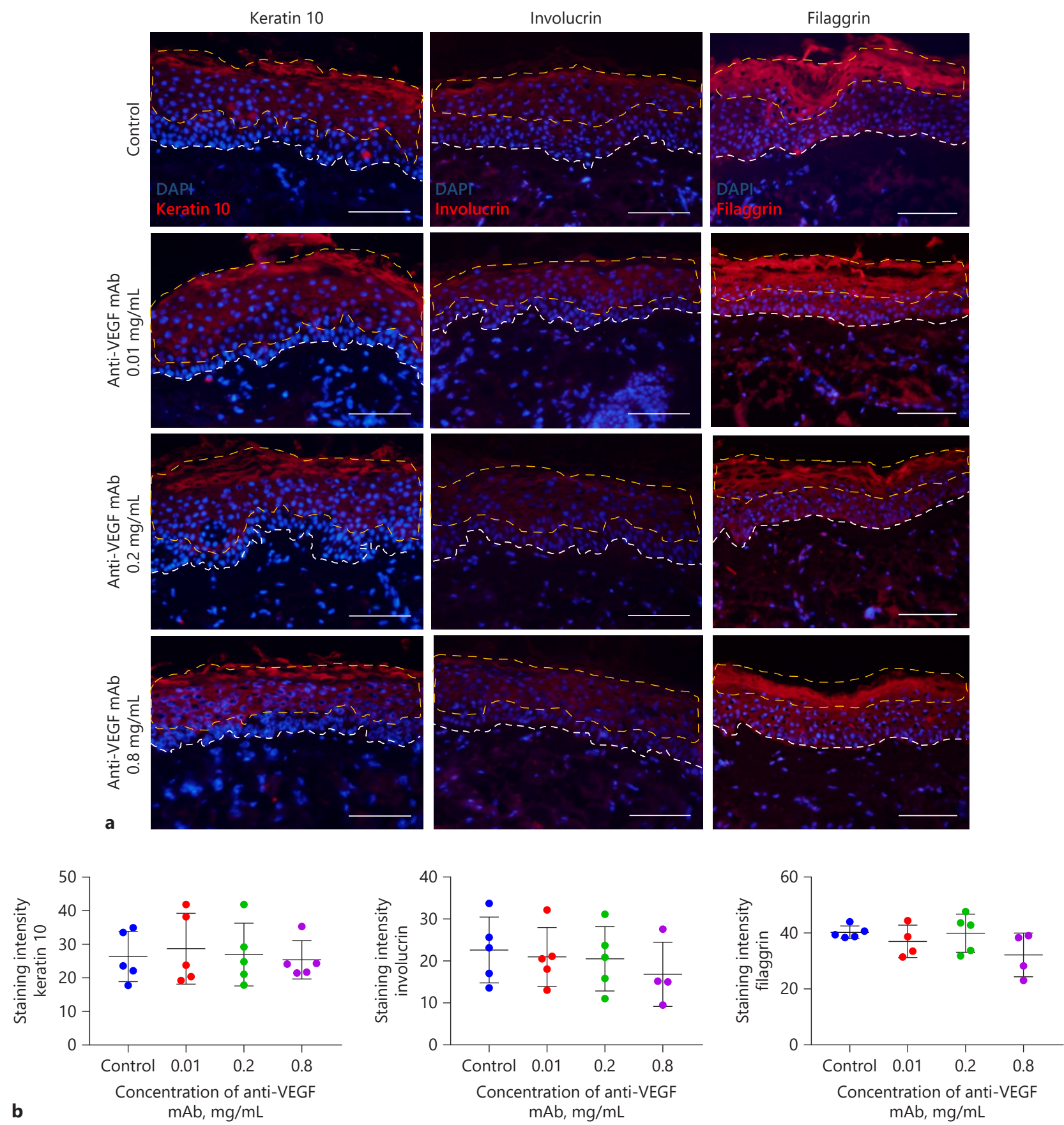

Fig. 2. Anti-VEGF mAb did not affect keratinocyte terminal differentiation in skin organ culture ex vivo. a The expression of markers of terminal differentiation keratin 10, involucrin, and filaggrin was studied in the upper layers of the epidermis (area surrounded by the yellow dotted line). DAPI was used for nuclear staining, and the white broken line indicates the epidermal-dermal junction. b Bevacizumab did not affect keratin 10, involucrin, or filaggrin expression in the epidermis. Number of independent experiments $n=5$ different donors (i.e., 1 punch per patient and per treatment group and at least 7 photomicrographs were analyzed per condition). VEGF, vascular endothelial growth factor; $\mathrm{mAb}$, monoclonal antibody. Scale bars $=100 \mu \mathrm{m}$. Data were analyzed with one-way ANOVA, mean \pm SD (Tukey's multiple comparison test). 
organ culture system are quiescent, as they would be, physiologically in healthy adult skin in vivo. In addition, our model facilitates the visualization of hyperproliferating endothelial cells and the effects of anti-VEGF agents on the cutaneous microvascular network. This is especially relevant for the study of skin diseases characterized by pathological angiogenesis, such as psoriasis. The assay also permits dissection of the mechanism of action by which anti-VEGF therapeutics induce endothelial cell apoptosis. Taken together, the pragmatic human skin organ culture assay reported here offers a valuable tool for future preclinical endothelial cell and microvascular network/anti-angiogenesis research in human skin.

\section{Acknowledgements}

We would like to acknowledge the British Skin Foundation, UK, for funding and acknowledge Dr. Gadea Mata for helping with the vascular morphometric analysis. This research was supported by the NIHR Manchester Biomedical Research Centre.

\section{Statement of Ethics}

Human skin samples were obtained through the Manchester Skin Health Biobank (19/NW/0082). Human tissue collection and handling was performed according to the "Human Tissue Act guidelines" with approval from the institutional research ethics committee. Written informed consent was obtained from the study volunteers, who were recruited from Salford Royal NHS Foundation Trust, Manchester, UK.

\section{Disclosure Statement}

The authors have no conflicts of interest to declare.

\section{Funding Sources}

This research study has been funded by the British Skin Foundation, UK.

\section{Author Contributions}

H.Y. and R.P. conceived the experiments. A.L.M. and J.H.S. performed the experiments. A.L.M., J.H.S., R.P. and H.Y. analyzed the data. H.Y. and R.P. acquired the funding. A.L.M. and D.R. acquired the tissue. All authors contributed to the writing/editing of the paper and approved the final manuscript.

\section{Availability of Data and Material}

Research data that are necessary to interpret the information presented here will be made available to any researcher, with minimal reuse restrictions.

\section{References}

1 Detmar M. Evidence for vascular endothelial growth factor (VEGF) as a modifier gene in psoriasis. J Invest Dermatol. 2004;122(1):xiv$\mathrm{xv}$.

2 Wong W. New connections: the duality of ROS in angiogenesis. Sci Signal. 2017;10(479): eaan64381.

3 Young HS, Summers AM, Read IR, Fairhurst, DA, Plant DJ, Campalani E, et al. Interaction between genetic control of vascular endothelial growth factor production and retinoid responsiveness in psoriasis. J Invest Dermatol. 2006;126(2):453-9.

4 Simons M, Gordon E, Claesson-Welsh L. Mechanisms and regulation of endothelial VEGF receptor signalling. Nat Rev Mol Cell Biol. 2016;17(10):611-25.

5 Hurwitz H, Fehrenbacher L, Novotny W, Cartwright T, Hainsworth J, Heim W, et al. Bevacizumab plus irinotecan, fluorouracil, and leucovorin for metastatic colorectal cancer. N Engl J Med. 2004;350(23):2335-42.
6 Akman A, Yilmaz E, Mutlu H, Ozdogan M. Complete remission of psoriasis following bevacizumab therapy for colon cancer. Clin Exp Dermatol. 2009;34(5):e202-4.

7 Datta-Mitra A, Riar NK, Raychaudhuri SP. Remission of psoriasis and psoriatic arthritis during bevacizumab therapy for renal cell cancer. Indian J Dermatol. 2014;59(6):632.

8 Stryker ZI, Rajabi M, Davis PJ, Mousa SA. Evaluation of angiogenesis assays. Biomedicines. 2019;7(2):37.

9 Zhang GY, Langan EA, Meier, NT, Funk W, Siemers F, Paus R. Thyroxine (T4) may promote re-epithelialisation and angiogenesis in wounded human skin ex vivo. PLoS One. 2019;14(3):e0212659.

10 Zhou L, Zhang X, Paus R, Lu Z. The renaissance of human skin organ culture: a critical reappraisal. Differentiation. 2018;104:22-35.

11 Eskens FA, Sleijfer S. The use of bevacizumab in colorectal, lung, breast, renal and ovarian cancer: where does it fit? Eur J Cancer. 2008; 44(16):2350-6.
12 Lu Z, Hasse S, Bodo E, Rose C, Funk W, Paus $\mathrm{R}$. Towards the development of a simplified long-term organ culture method for human scalp skin and its appendages under serumfree conditions. Exp Dermatol. 2007;16(1): $37-44$.

13 Nasir NAM, Paus R, Ansell DM. Fluorescent cell tracer dye permits real-time assessment of re-epithelialization in a serum-free ex vivo human skin wound assay. Wound Repair Regen. 2019;27(1):126-33.

14 Philpott MP, Sanders D, Westgate GE, Kealey T. Human hair growth in vitro: a model for the study of hair follicle biology. J Dermatol Sci. 1994;7(Suppl 1):S55-72.

15 Wang Y, Fei D, Vanderlaan M, Song A. Biological activity of bevacizumab, a humanized anti-VEGF antibody in vitro. Angiogenesis. 2004;7(4):335-45.

16 Falk AT, Barrière J, François E, Follana P. Bevacizumab: a dose review. Crit Rev Oncol Hematol. 2015;94(3):11-22. 
17 Panoilia E, Schindler E, Samantas E, Aravantinos G, Kalofonos HP, Christodoulou C, et al. A pharmacokinetic binding model for bevacizumab and VEGF165 in colorectal cancer patients. Cancer Chemother Pharmacol. 2015;75(4):791-803.

18 Peraza MA, Rule KE, Shiue MHI, Finch GL, Thibault S, Brown PR, et al. Nonclinical assessments of the potential biosimilar PF06439535 and bevacizumab. Regul Toxicol Pharmacol. 2018;95:236-43.

19 Grothey A, Galanis, E. Targeting angiogenesis: progress with anti-VEGF treatment with large molecules. Nat Rev Clin Oncol. 2009; 6(9):507-18.
20 Dostalek M, Gardner I, Gurbaxani BM, Rose RH, Chetty M. Pharmacokinetics, pharmacodynamics and physiologically-based pharmacokinetic modelling of monoclonal antibodies. Clin Pharmacokinet. 2013;52(2):83-124.

21 Jean J, Leroy M, Duque-Fernandez A, Bernard G, Soucy J, Pouliot R. Characterization of a psoriatic skin model produced with involved or uninvolved cells. J Tissue Eng Regen Med. 2015;9(7):789-98.
22 Gudjonsson JE, Ding J, Li X, Nair RP, Tejasvi T, Qin ZS, et al. Global gene expression analysis reveals evidence for decreased lipid biosynthesis and increased innate immunity in uninvolved psoriatic skin. J Invest Dermatol. 2009;129(12):2795-804.

23 Batista MD, Tincati C, Milush JM, Ho EL, Ndhlovu LC, York VA, et al. CD57 expression and cytokine production by $\mathrm{T}$ cells in lesional and unaffected skin from patients with psoriasis. PLoS One. 2013;8(2):e52144.

24 Varricchi G, Granata F, Loffredo S, Genovese A, Marone G. Angiogenesis and lymphangiogenesis in inflammatory skin disorders. J Am Acad Dermatol. 2015;73(1):144-53. 